\title{
Effect of sildenafil on digital ulcers in systemic sclerosis: analysis from a single centre pilot study
}

\author{
Claudia S Brueckner, ${ }^{1}$ Mike 0 Becker, ${ }^{1}$ Thomas Kroencke, ${ }^{2}$ Doerte Huscher, ${ }^{3}$ \\ Hans Ulrich Scherer, ${ }^{1}$ Margitta Worm, ${ }^{4}$ Gerd Burmester, ${ }^{1}$ Gabriela Riemekasten ${ }^{1}$
}

${ }^{1}$ Clinic of Rheumatology and Clinical Immunology, Charité Universitätsmedizin Berlin, Germany

${ }^{2}$ Clinic of Radiology and Interventional Radiology, Charité Universitätsmedizin Berlin, Germany

${ }^{3}$ German Rheumatology Research Centre, Berlin,

Germany

${ }^{4}$ Clinic of Dermatology, Venerology, and Allergology, Charité Universitätsmedizin Berlin, Germany

\section{Correspondence to}

Dr Gabriela Riemekasten, Clinic of Rheumatology and Clinical Immunology, Charitéplatz 1 , 10117 Berlin, Germany; gabriela.Riemekasten@charite. de

Accepted 3 October 2009

\begin{abstract}
Objective In this pilot study, the effect of sildenafil on digital ulcer (DU) healing and related clinical symptoms was analysed.

Methods A total of 19 patients with systemic sclerosis (SSc) were treated with maximally tolerated sildenafil doses up to 6 months. Primary outcome was the healing of DUs. Changes in other clinical symptoms were also evaluated.

Results In all, 49 DUs were present at baseline; this decreased to 17 ulcers $(p<0.001)$ at the end of sildenafil treatment. Furthermore, the visual analogue scale (VAS) score for Raynaud's phenomenon (RP), pain and activity improved $(p=0.003, p=0.002$ and $p=0.05$, respectively). A total of 9 patients developed 12 new DUs during sildenafil treatment.

Conclusions This study indicates an effect of sildenafil on DU healing in patients with SSc and an improvement of RP and associated symptoms that should be validated in controlled studies.
\end{abstract}

\section{INTRODUCTION}

Systemic sclerosis (SSc) is a devastating disease with a high impact on quality of life and prognosis. Vasculopathy is an early and prominent feature and is reflected by Raynaud's phenomenon (RP), the presence of digital ulcers (DUs) and pulmonary arterial hypertension $\left(\mathrm{PAH}^{1}\right)$. Recent therapeutic advances suggest common pathogenic mechanisms and a role for endothelin receptor activation in SSc-associated vasculopathy. ${ }^{2}$ However, endothelin receptor blockers such as the dual endothelin receptor type A/B blocker bosentan are effective in the prevention of DUs, but are not able to heal DUs, indicating different mechanisms for prevention and ulcer healing. ${ }^{3}$ Intravenous iloprost is often used for the treatment of DUs, but its effect on the prevention of DUs has not been studied to date. There is also a subgroup of patients that do not respond to iloprost treatment. ${ }^{4}$

Reduced levels of nitric oxide have also been proposed to play a role in the pathogenesis of vascular disease in systemic sclerosis. ${ }^{5}$ Therefore, sildenafil as a selective inhibitor of cyclic guanosine monophosphate (cGMP)-specific phosphodiesterase type 5 and potent agent to increase the endogenous NO levels is an attractive candidate for the treatment of SSc-associated vasculopathy. Indeed, sildenafil is approved by the US Food and Drug Administration and the European Medicines Agency for the treatment of PAH. The highest dose of sildenafil recommended for the treatment of PAH is $60 \mathrm{mg}$ daily; however, several studies suggest a higher effect by increasing the dose. ${ }^{6}$ 7 In a placebo-controlled crossover study, $50 \mathrm{mg}$ sildenafil twice daily for 4 weeks was shown to be effective for the treatment of RP. ${ }^{8}$ Furthermore, some case reports have suggested an effect on DU healing in SSc. ${ }^{10}$ Therefore, we conducted a pilot study analysing the effect of sildenafil on healing of SSc-associated DUs refractory to other treatments.

\section{PATIENTS AND METHODS}

A total of 19 patients with SSc (mean age 51 years) fulfilling the American College of Rheumatology (ACR) criteria for systemic sclerosis, assessed according to the European League Against Rheumatism (EULAR) Scleroderma Trials And Research (EUSTAR) criteria, ${ }^{11}$ and experiencing severe rhagades $(n=1)$ or digital ulcers $(n=18)$ refractory to treatment were treated with the maximally tolerated sildenafil doses (up to $150 \mathrm{mg}$ ) for a maximum of 6 months. Inclusion criteria were stable treatment with vasoactive and immunosuppressive drugs for 3 months. Current smokers, patients with gangrene, a history of gastric ulcers during the last 3 months, cardiac ejection fraction below $25 \%$, patients with severe organ involvement or other uncontrolled diseases were excluded. DUs were defined as a loss of epidermis and dermis. The demographic data are shown in table 1.

The study was conducted between 2004 and 2007. Two patients were treated outside of the study with the intention to heal digital ulcers refractory to other treatments. The other 17 patients were treated as part of a prospective, open, single-centre study. Here, the primary outcome parameter was DU healing. Secondary end points were effects on Raynaud's phenomenon by using a visual analogue scale (VAS), the prevention of new ulcers, changes in VAS scores for pain, activity and experiencing ulcers, the improvement of rhagades, Scleroderma Health Assessment Questionnaire (SHAQ) indices and the occurrence of sildenafil-related adverse effects. Drop out criteria/final end points were any changes in the immunosuppressive treatments, no response on ulcer healing within 1 month, or escalation of medication with vascular effects. Relevant macrovasculopathy responsible for DUs was excluded by angiography or duplex sonography. ${ }^{12}$ Nine patients treated for 6 months by sildenafil received a second angiography.

The study was approved by the local ethical committee (SDN-D-002G) and published as a clinical trial (NCT00624273). Patients signed an individual informed consent. online under the BMJ Journals unlocked scheme, see http:// ard.bmj.com/info/unlocked.dtl 
Table 1 Patient characteristics and effects of treatments including previous and concomitant treatments

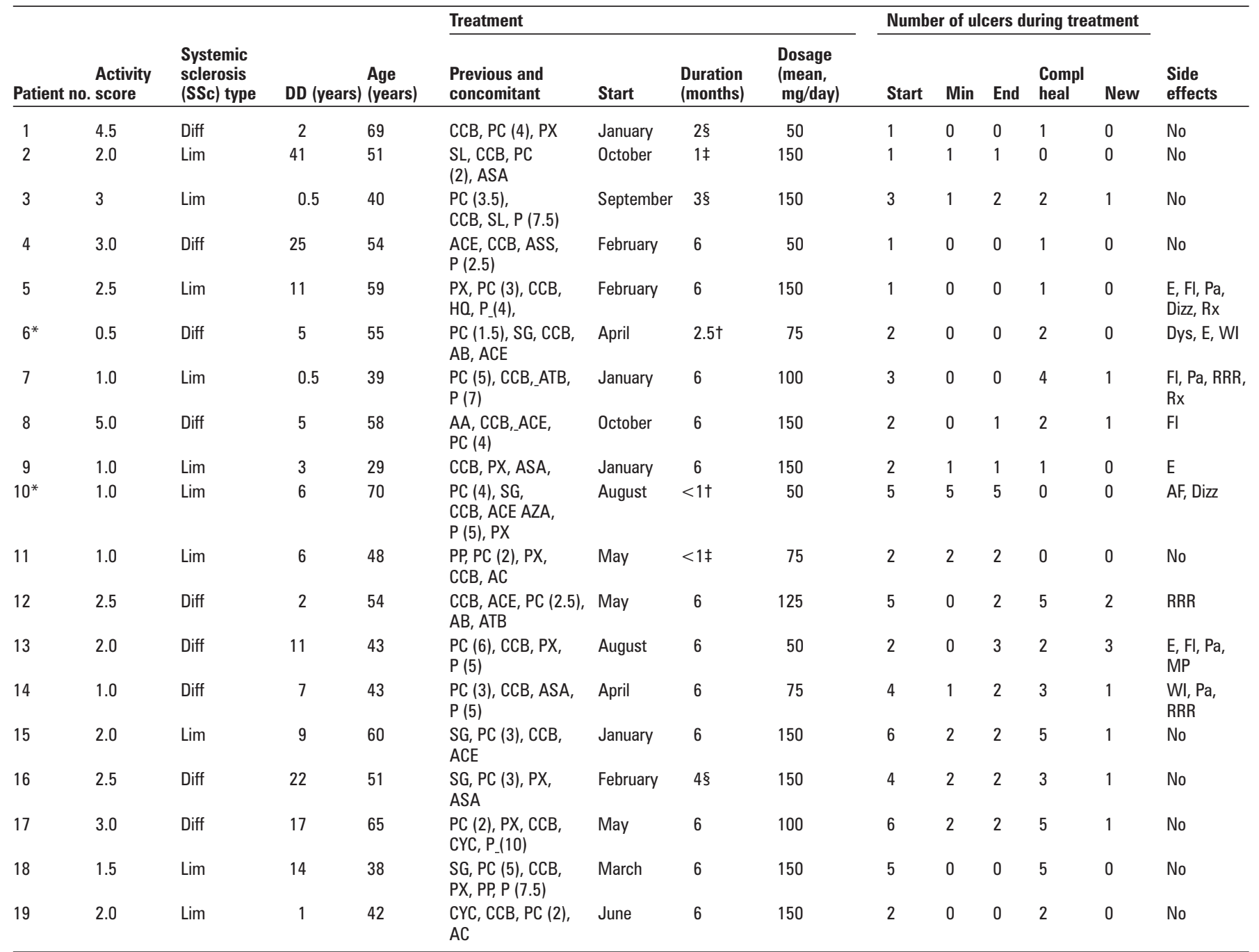

SSc type: lim, limited; diff, diffuse.

*Indicates male patients.

tIndicates cessation of treatment due to side effects.

fIndicates cessation of treatment due to other events with possible impact on the effect of treatment (ulcer infection, cyclophosphamide use).

§Indicates patients with development of new digital ulcer or calcinosis and no significant improvement by treatment.

$A A$, autoamputation; $A B, \alpha$ blocker; $A C$, anticoagulation; $A C E$, angiotensin converting enzyme inhibitor; $A F$, atrial fibrillation; $A S A$, acetyl salicylic acid; $A T B$, angiotensin recepto

blocker; AZA, azathioprine; BWI, body weight increase; CCB, calcium channel blockers; compl heal, number of ulcers completely healed; CYC, cyclophosphamide; DD, disease duration;

Dizz, dizziness; Dys, dyspnoea; E, Oedema; Fl, flush; HQ, hydroxycloroquine; MP, muscle pain; P, prednisone (mg/day); Pa, palpitations; PC, intravenous prostacyclin (weeks with

maximally tolerated dose for $6 \mathrm{~h} /$ day); PP, plasmaphereses; PX, pentoxyphylline; RRR, reduction of RR (arterial hypertension) medication; Rx, reflux; SG, surgery; SL, sympathicolysis.

\section{Statistics}

A paired Wilcoxon test was used to identify the effect of sildenafil on digital ulcers and VAS for clinical symptoms. Graph Pad Prism V.3.02 (Graph Pad Software, San Diego, California, USA) for Microsoft Windows was used.

\section{RESULTS}

Three patients did not receive sildenafil for more than 1 month due to myositis requiring immunosuppression $(n=1)$, the reoccurrence of atrial fibrillation $(\mathrm{n}=1)$ and infection of an ulcer with a multiresistant staphylococcus $(\mathrm{n}=1)$, and were excluded from further analyses. In the remaining 16 patients, the mean sildenafil dose was $114 \mathrm{mg} /$ day, and the mean duration of treatment was 5.2 months. At baseline, 49 ulcers were present (mean 3.1/ patient). Most patients showed a rapid response reaching a minimum of DUs within 3 months without differences in SSc subtype (figure 1A,B; figure 2). The minimal number of DUs was achieved in each patient at different time points; minimally nine
DUs were present (mean 0.6 ulcers/patients, $\mathrm{p}<0.001$ compared to initial numbers, table 1 ). At the end of sildenafil treatment, 17 digital ulcers were detectable (mean 1.1/patient), which was different from the number of ulcers at baseline $(p<0.001$, table 1$)$.

During sildenafil treatment, 9 patients developed 12 new DUs resulting in treatment being stopped in 3 patients. In one of these patients a diagnosis of calcinosis was made. A total of 12 patients were treated for 6 months. VAS scores for RP, pain and activity significantly improved $(p=0.003, p=0.002$ and $p=0.05$, respectively) with sildenafil treatment (figure $1 \mathrm{C}-\mathrm{F}$ ). There was also a tendency for changes in the disability score due to the DUs $(p=0.08)$. SHAO Disability Index (SHAQ-DI) scores available from 11 patients improved from a mean of 0.6 (SD 0.52 ) to 0.42 (SD 0.32) but failed to reach statistical significance $(p=0.14)$. In the nine patients that received angiography before and after treatment, one responder showed an improvement of vascular pathology (not shown). Severe rhagades disappeared by sildenafil in the one affected patient. Of the 14 patients with 
A
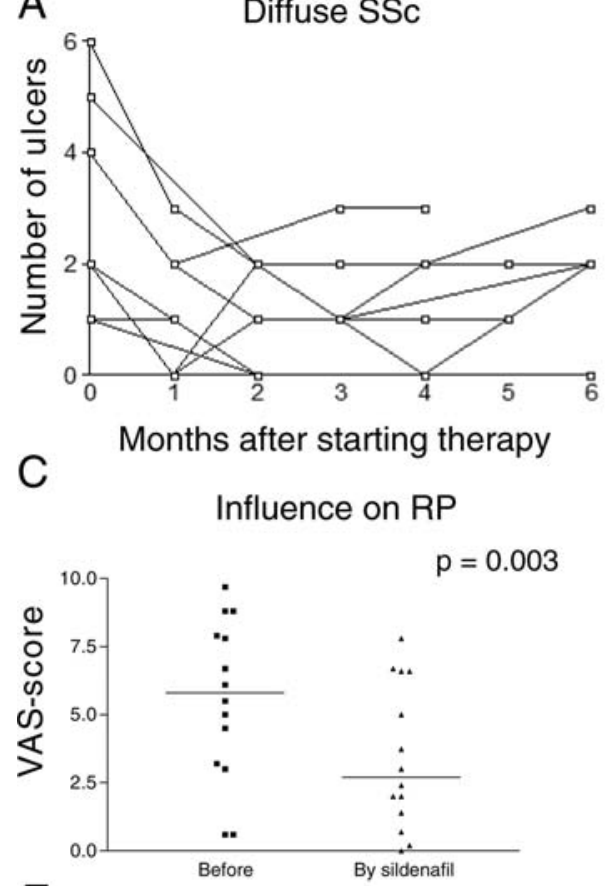

$\mathrm{E}$

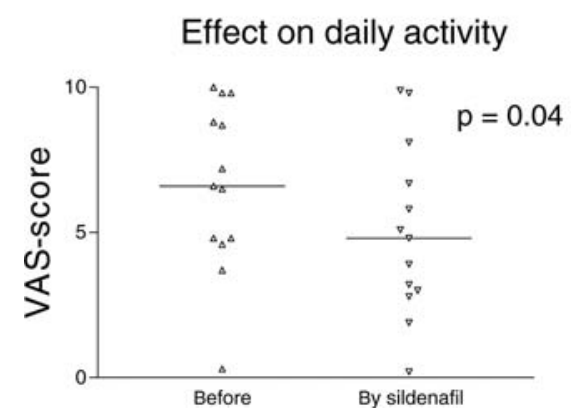

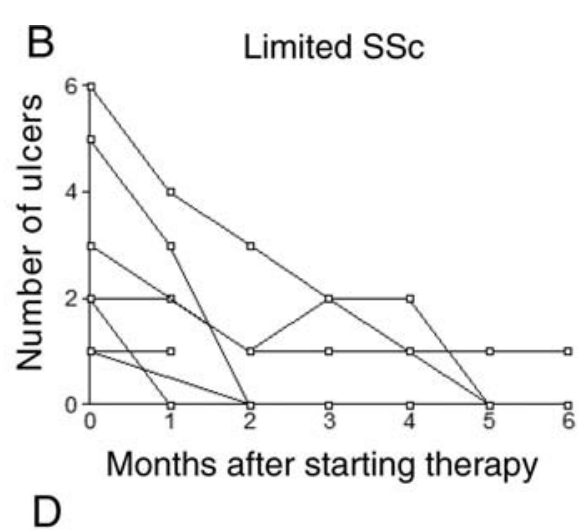
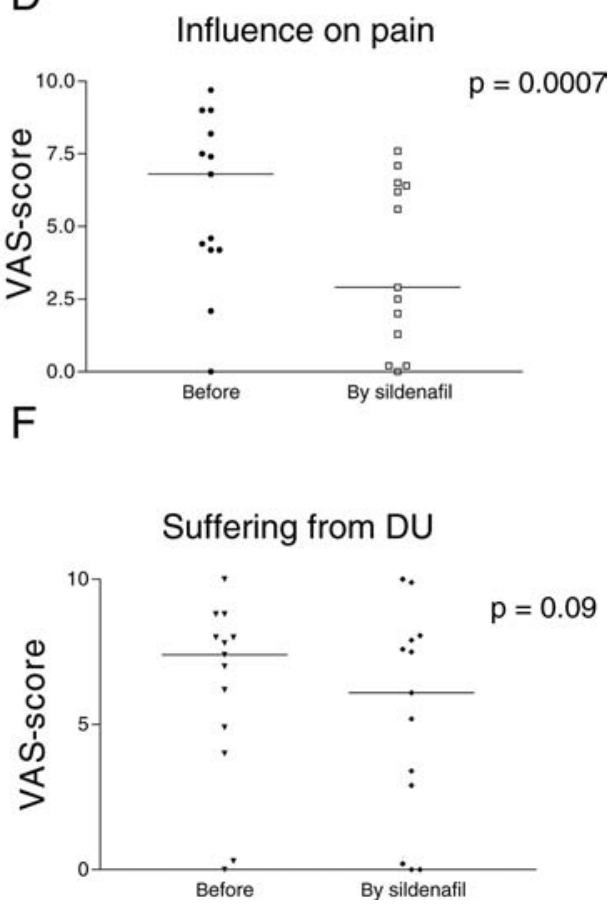

Figure 1 Effect of sildenafil treatment on the number of digital ulcers (DU) in patients with (A) diffuse and (B) limited systemic sclerosis (SSc) during 6 months of treatment, on (C) Raynaud's phenomenon (RP), (D) burden of pain, (E) daily activity and (F) on disease burden due to ulcers measured by visual analogue scale (VAS). A paired Wilcoxon test was used to calculate statistical significance; the median is illustrated as a horizontal line.

follow-up data available, 7 patients developed 15 DUs within 3 months, and 3 patients took sildenafil outside of the study.

Mild side effects were common and were present in 9 out of 19 patients, but resulted in a discontinuation of the treatment only in the 2 male patients (table 1). In three patients with arterial hypertension, the antihypertensive treatment was reduced during sildenafil treatment.

\section{DISCUSSION}

In a previous single-centre placebo-controlled crossover study, sildenafil was shown to improve the capillary blood flow and RP. As suggested by a few patients with DUs, sildenafil could provide a useful therapeutic approach for DU healing. In this prospective, open pilot study, maximally tolerated sildenafil doses seem to be effective in DU healing. The effect was rapid, and maximum benefit was reached in the first few months. Furthermore, the study confirmed the beneficial effect of sildenafil on RP and microvasculopathy. DUs are a very severe complication with a high impact on the quality of life in patients with SSc. At present, there is no approved treatment for the healing of DUs. The dual endothelin-1 receptor antagonist has failed to be effective in ulcer healing. ${ }^{3}$ However, different studies have shown the effect of bosentan in preventing the development of new DUs. ${ }^{3}{ }^{13}$ In the present study, sildenafil shows benefit in DU healing, confirming case reports and initial observations in which sildenafil was given only for a few weeks. ${ }^{8}$ In our study, patients were treated for up to 6 months. Within this period, a significant proportion of patients developed new DUs, suggesting a failure of sildenafil to prevent new structural defects. Furthermore, only one patient had mild improvement by angiography. As already suggested by others, different pathogenetic mechanisms could be responsible for prevention or DU healing. ${ }^{2} 14$ Therefore, as also discussed for the treatment of $\mathrm{PAH}$, combination treatments targeting different pathogenic mechanisms could provide a more valuable and effective tool for further treatments.

A major limitation of this study is the lack of a placebo group. When compared to the placebo group of the "Rheumatoid Arthritis PreventIon of structural Damage 1' (RAPID 1) study in which about $10 \%$ of ulcers healed per month, ${ }^{3}$ the number of ulcers healed was higher in our sildenafil group. However, direct comparison with other trial data provide only limited information due to differing study designs. Furthermore, most of the patients treated here had been refractory to previous treatments. 

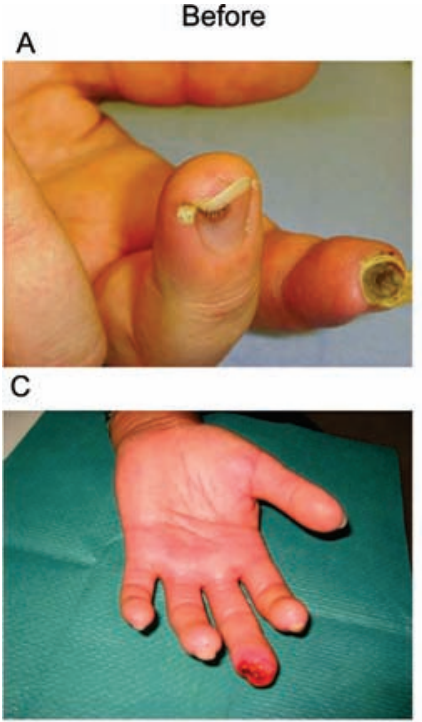

E

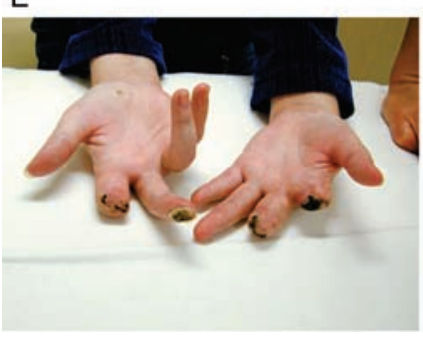

After sildenafil

B

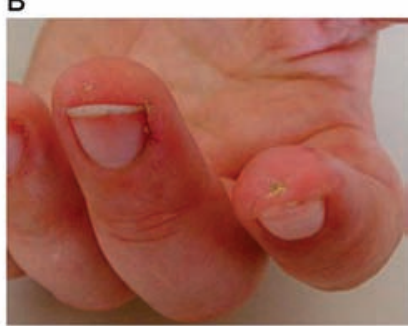

D

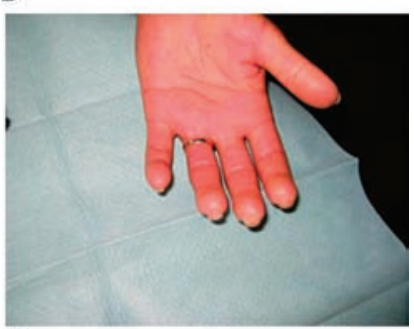

F

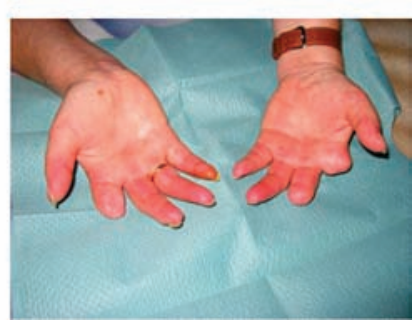

Figure 2 Digital ulcer healing by sildenafil treatment as shown for three different patients. Digital ulcers before treatment are shown in $(A),(C)$ and $(D) ;(B),(D)$ and $(F)$ show the effects of sildenafil treatment; (E) and (F) come from a patient refractory to continuous intravenous iloprost treatment over 2 weeks requiring amputation and showing insufficient wound healing. Within a few days, the patient remarked at finger rewarming and subsequently progressive wound healing.

Therefore, our study population could represent a subgroup of patients with SSc truly responding to sildenafil treatment.

For treatment of PAH or erectile dysfunction, lower doses of sildenafil are usually used. In our study, maximally tolerated doses were given for the treatment of DUs. Treatment was well tolerated and side effects were not dose related. The two male patients who discontinued the drug due to side effects received low sildenafil doses $(2 \times 25 \mathrm{mg} /$ day $)$, suggesting a higher sensitivity to the drug other than the use of unconventionally high doses. The incidence of peripheral oedema, the presence of dyspnoea and reoccurrence of atrial fibrillation suggest increased blood flow and fluid overload in few patients. This remains to be further evaluated since phosphodiesterase- 5 inhibitors were also successfully used to treat pulmonary oedema. ${ }^{15}$

In conclusion, this pilot study indicates beneficial and rapid effects in the treatment of DUs. Moreover, sildenafil decreases the burden of RP and associated symptoms. The data encourages controlled studies, and combining a preventive as well as a curative treatment would be a valuable approach to treat DUs in patients with SSc.

Funding The study was supported by an unrestricted grant from Pfizer. Furthermore, the study was supported by the German Network for Systemic Sclerosis (DNSS) as well as by the EUSTAR network for assessment strategies.

Competing interests GR received lecturer's fees from Encysive, Actelion, GSK and Pfizer.

Ethics approval This study was conducted with the approval of the local ethical committee (Charité Berlin).

Provenance and peer review Not commissioned; externally peer reviewed.

Patient consent Obtained.

\section{REFERENCES}

1. Sunderkötter C, Riemekasten G. Pathophysiology and clinical consequences of Raynaud's phenomenon related to systemic sclerosis. Rheumatology (Oxford) 2006;45(Suppl 3):iii33-5.

2. Abraham D, Distler 0 . How does endothelial cell injury start? The role of endothelin in systemic sclerosis. Arthritis Res Ther 2007;9(Suppl 2):S2.

3. Korn JH, Mayes M, Matucci Cerinic M, et al. Digital ulcers in systemic sclerosis: prevention by treatment with bosentan, an oral endothelin receptor antagonist. Arthritis Rheum 2004;50:3985-93.

4. Kawald A, Burmester GR, Huscher D, et al. Low versus high-dose iloprost therapy over 21 days in patients with secondary Raynaud's phenomenon and systemic sclerosis: a randomized, open, single-center study. J Rheumatol 2008;35:1830-7.

5. Freedman RR, Girgis R, Mayes MD. Acute effect of nitric oxide on Raynaud's phenomenon in scleroderma. Lancet 1999;354:739.

6. Garg N, Sharma MK, Sinha N. Role of oral sildenafil in severe pulmonary arterial hypertension: clinical efficacy and dose response relationship. Int J Cardiol 2007:120:306-13.

7. Badesch DB, Hill NS, Burgess G, et al. Sildenafil for pulmonary arterial hypertension associated with connective tissue disease. J Rheumatol 2007;34:2417-22.

8. Fries R, Shariat K, von Wilmowsky H, et al. Sildenafil in the treatment of Raynaud's phenomenon resistant to vasodilatory therapy. Circulation 2005;112:2980-5.

9. Colglazier CL, Sutej PG, O'Rourke KS. Severe refractory fingertip ulcerations in a patient with scleroderma: successful treatment with sildenafil. J Rheumatol 2005;32:2440-2.

10. Friedrichson $\mathbf{E}$, Rehberger P, Fuhrmann JT, et al. [Fast and efficient healing of scleroderma-associated acral ulcers with sildenafill. Hautarzt 2008;59:230-2.

11. Walker UA, Tyndall A, Czirják L, et al. Clinical risk assessment of organ manifestations in systemic sclerosis: a report from the EULAR Scleroderma Trials And Research group database. Ann Rheum Dis 2007;66:754-63.

12. Stoeckelhuber BM, Suttmann I, Stoeckelhuber M, et al. Comparison of the vasodilating effect of nitroglycerin, verapamil, and tolazoline in hand angiography. $J$ Vasc Interv Radiol 2003;14:749-54.

13. García de la Peña-Lefebvre $\mathbf{P}$, Rodríguez Rubio $\mathrm{S}$, Valero Expósito $\mathrm{M}$, et al. Long-term experience of bosentan for treating ulcers and healed ulcers in systemic sclerosis patients. Rheumatology (Oxford) 2008;47:464-6.

14. Guiducci S, Distler O, Distler JH, et al. Mechanisms of vascular damage in SSc-implications for vascular treatment strategies. Rheumatology (Oxford) 2008;47(Suppl 5):v18-20.

15. Maggiorini M, Brunner-La Rocca HP, Peth S, et al. Both tadalafil and dexamethasone may reduce the incidence of high-altitude pulmonary edema: a randomized trial. Ann Intern Med 2006;145:497-506. 